\title{
A LITTLEWOOD AND PALEY-TYPE INEQUALITY ON THE BALL
}

\author{
Jun Soo Choa and Hong Oh KIM
}

\begin{abstract}
A multi-dimensional analogue of a well known inequality of Littlewood and Paley is obtained on the ball.
\end{abstract}

\section{INTRODUCTION AND STATEMENT OF THE RESULT}

A well known inequality of Littlewood and Paley states that (see [2, 3]) : if $2 \leqslant$ $p<\infty$ and $f$ is a function, analytic in the unit disk, $D$, which belongs to the Hardy space $H^{p}(D)$, then there exists a positive constant $C=C(p)$ such that

$$
\int_{D}\left(1-|z|^{2}\right)^{p-1}\left|f^{\prime}(z)\right|^{p} d A(z) \leqslant C\|f\|_{H^{p}(D)}^{p}
$$

where $A$ is the normalised area measure on $D$.

Recently Luecking [3] gave a new proof of this result, which motivates our work. In this note we shall extend the result of Littlewood and Paley to the multi-dimensional case. To state our result, we need some notation and definitions.

Let $B$ be the unit ball of the $n$-dimensional complex space $\mathbf{C}^{n}$. (Hereafter $n$ will be fixed.) The letter $V$ stands for the normalised Lebesgue measure on $B$ so that $V(B)=1$, while $\sigma$ is the normalised surface area measure on its boundary $S$.

For $0<p<\infty$, the Hardy space $H^{p}(B)$ is defined to be the set of all analytic functions $f$ on $B$ for which

$$
\|f\|_{H^{p}(B)}^{p}=\sup _{0<r<1} \int_{S}|f(r \xi)|^{p} d \sigma(\xi)<\infty .
$$

It is well known [4] that if $f \in H^{p}(B)$ for $0<p<\infty$, then the radial limit $f^{*}(\xi)=$ $\lim _{r \rightarrow 1} f(r \xi)$ exists for $[\sigma]$-almost all $\xi \in S$ and $\|f\|_{B(B)}^{p}=\int_{S}\left|f^{*}(\xi)\right|^{p} d \sigma(\xi)$. We denote $f \in H^{p}(B)$ and its radial limit function $f^{*}$ by the same latter $f$.

The result in this note is formulated as follows :

Received 7th December, 1994.

This research was partially supported by KOSEF and TGRC.

Copyright Clearance Centre, Inc. Serial-fee code: 0004-9729/94 \$A2.00+0.00. 
Theorem. Let $2 \leqslant p<\infty$. Then there exists a positive constant $C=C(p)$ such that

$$
\int_{B}\left(1-|z|^{2}\right)^{p / 2-1}\left(|\nabla f(z)|^{2}-|\mathcal{R} f(z)|^{2}\right)^{p / 2} d V(z) \leqslant C\|f\|_{B^{p}(B)}^{p}
$$

for all $f \in H^{p}(B)$.

Here and elsewhere, $\nabla f(z)=\left(\frac{\partial f}{\partial z_{1}}, \cdots, \frac{\partial f}{\partial z_{n}}\right)$ denotes the complex gradient of $f$ and $\mathcal{R} f(z)=\sum_{j=1}^{n} z_{j} \frac{\partial f}{\partial z_{j}}$ denotes the radial derivative of $f$.

\section{Preliminaries}

2.1 AUtomorphisms of $B$. For $z, w \in \mathbf{C}^{n}$ let $\langle z, w\rangle=\sum_{j=1}^{n} z_{j} \bar{w}_{j}$ denote the complex inner product on $\mathbf{C}^{n}$ and $|z|=\langle z, z\rangle^{1 / 2}$. For $a, z \in B,(a \neq 0)$ define

$$
\varphi_{a}(z)=\frac{a-P_{a} z-\left(1-|a|^{2}\right)^{1 / 2} Q_{a} z}{1-\langle z, a\rangle}
$$

where $P_{a} z=\langle z, a\rangle a /|a|^{2}$ and $Q_{a} z=z-P_{a} z$. For $a=0$ we let $\varphi_{0}(z)=-z$. Then $\varphi_{a}(0)=a$ and $\left(\varphi_{a} \circ \varphi_{a}\right)(z)=z$ for all $z \in B$. Thus $\varphi_{a} \in$ Aut $(B)$, the group of all automorphsims of $B$. Furthermore each $\psi \in \operatorname{Aut}(B)$ has a unique representation $\psi=U \circ \varphi_{a}$ for some $a \in B$ and some unitary transformation $U$ on $\mathbf{C}^{n}$. The following two properties of $\varphi_{a}$ are found in Rudin [4, Section 2.2].

$$
\begin{aligned}
& 1-\left|\varphi_{a}(z)\right|^{2}=\frac{\left(1-|a|^{2}\right)\left(1-|z|^{2}\right)}{|1-\langle z, a\rangle|^{2}} \\
& J_{R} \varphi_{a}(z)=\left(\frac{1-|a|^{2}}{|1-\langle z, a\rangle|^{2}}\right)^{n+1}
\end{aligned}
$$

where $J_{R} \varphi_{a}$ is the real Jacobian of $\varphi_{a}$.

2.2 Invariant Laplacian. For $f \in C^{2}(B)$ and $a \in B$, we define

$$
(\widetilde{\Delta} f)(a)=\frac{1}{n+1} \Delta\left(f \circ \varphi_{a}\right)(0)
$$

where $\triangle=4 \sum_{j=1}^{n} \frac{\partial^{2}}{\partial a_{j} \partial \bar{a}_{j}}$ is the ordinary Laplacian. The operator $\widetilde{\Delta}$ is invariant under Aut $(B)$, that is, $(\widetilde{\Delta}) \circ \psi=\widetilde{\triangle}(f \circ \psi)$ for all $\psi \in \operatorname{Aut}(B)$. For this reason, $\widetilde{\Delta}$ is 
called the invariant Laplacian. In terms of partial derivatives, the invariant Laplacian $\widetilde{\Delta}$ is computed as follows:

$$
(\widetilde{\Delta} f)(a)=\frac{4}{n+1}\left(1-|a|^{2}\right) \sum_{j, k=1}^{n}\left(\delta_{j k}-a_{j} \bar{a}_{k}\right) \frac{\partial^{2} f(a)}{\partial a_{j} \partial \bar{a}_{k}},
$$

where $\delta_{j k}$ is the Kroneker symbol. See [4, Section 4.1] for details.

2.3 GREen's FUnCTIONS FOR $\widetilde{\triangle}$. For each $a \in B$, we define the Green's funtion $G(z, a)$ for $\widetilde{\triangle}$ on $B$ by

$$
G(z, a)=g\left(\varphi_{a}(z)\right)
$$

where $g(z)=(n+1) /(2 n) \int_{|z|}^{1}\left(1-t^{2}\right)^{n-1} t^{-2 n+1} d t$. See [5] for more information about Green's functions.

\section{Proof of the theoreM}

We begin with the following Littlewood-Paley type identity whose proof is a slight modification of ones given in $[1$, Theorem $A]$, and so we state it without proof.

Lemma 1. If $2 \leqslant p<\infty$ and $h \in H^{p}(B)$, then

$$
\int_{S}|h|^{p} d \sigma=|h(0)|^{p}+\int_{B}\left(\widetilde{\Delta}|h|^{p}\right)(z) g(z) d \tau(z)
$$

where $g(z)$ is the function as defined in Section 2 and $d \tau(z)=\left(1-|z|^{2}\right)^{-(n+1)} d V(z)$.

Lemma 2. Suppose $2 \leqslant p<\infty$ and $a \in B$. Then

$$
\left(\widetilde{\Delta}|f|^{p}\right)(a)=\frac{p^{2}}{4}|f(a)|^{p-2}\left(\widetilde{\Delta}|f|^{2}\right)(a)
$$

for every analytic function $f$ on $B$.

Proof: For $f$ analytic on $B$, using the property (3), we have

$$
\left(\widetilde{\Delta}|f|^{2}\right)(a)=\frac{4}{n+1}\left(1-|a|^{2}\right)\left(|\nabla f(a)|^{2}-|\mathcal{R} f(a)|^{2}\right) .
$$

Replacing $f$ by $f^{p / 2}$ in (6) (here $f^{p / 2}=\exp ((p / 2) \log f)$ is the principal branch), we get

$$
\left(\widetilde{\Delta}|f|^{p}\right)(a)=\frac{4}{n+1}\left(1-|a|^{2}\right)\left(\left|\nabla f^{p / 2}(a)\right|^{2}-\left|\mathcal{R} f^{p / 2}(a)\right|^{2}\right) .
$$


A little computation shows that

$$
\left|\nabla f^{p / 2}(a)\right|^{2}=\frac{p^{2}}{4}|f(a)|^{p-2}|\nabla f(a)|^{2}
$$

and

$$
\left|\mathcal{R} f^{\frac{p}{2}}(a)\right|^{2}=\frac{p^{2}}{4}|f(a)|^{p-2}|\mathcal{R} f(a)|^{2} .
$$

By inserting these into (7) we obtain

$$
\left(\tilde{\triangle}|f|^{p}\right)(a)=\frac{p^{2}}{n+1}\left(1-|a|^{2}\right)|f(a)|^{p-2}\left(|\nabla f(a)|^{2}-|\mathcal{R} f(a)|^{2}\right)
$$

which gives the desired conclusion.

LEMMA 3. If $2 \leqslant p<\infty$ and $f \in H^{p}(B)$, then there exists a positive constant $C=C(p)$ such that

$$
|\nabla f(0)|^{p} \leqslant C\left(\|f\|_{B p(B)}^{p}-|f(0)|^{p}\right)
$$

Proof: Suppose $f \in H^{p}(B)$ with $2 \leqslant p<\infty$. Then by the reproducing property we have

$$
f(z)-f(0)=\int_{S} \frac{f(\xi)-f(0)}{(1-\langle z, \xi\rangle)^{n}} d \sigma(\xi) .
$$

Differentiation under the integral sign gives

$$
\frac{\partial f}{\partial z_{j}}(0)=n \int_{S} \xi_{j}(f(\xi)-f(0)) d \sigma(\xi), 1 \leqslant j \leqslant n .
$$

It follows that

$$
\begin{aligned}
|\nabla f(0)|^{2} & \leqslant n^{2} \int_{S}|f(\xi)-f(0)|^{2} d \sigma(\xi) \\
& =n^{2}\left(\|f\|_{B^{2}(B)}^{2}-|f(0)|^{2}\right) .
\end{aligned}
$$

Without loss of generality, we may assume that $f(0)=0$ or $|f(0)|=1$.

In the first case, we clearly obtain

$$
|\nabla f(0)|^{p} \leqslant n^{p}\left(\|\left. f\right|_{H^{p}(B)} ^{p}-|f(0)|^{p}\right)
$$

because $\|f\|_{H^{2}(B)} \leqslant\|f\|_{H^{p}(B)}$ for $p \geqslant 2$. 
We next consider the case $|f(0)|=1$. A simple calculation shows that

$$
(x-1)^{p / 2} \leqslant x^{p / 2}-1 \text { for } x \geqslant 1
$$

Replacing $x$ by $\|f\|_{B^{p}(B)}^{2}$ and 1 by $|f(0)|^{2}$ in (9), we deduce that

$$
\begin{aligned}
|\nabla f(0)|^{p} & \leqslant n^{p}\left(\|f\|_{H^{p}(B)}^{2}-|f(0)|^{2}\right)^{p / 2} \\
& \leqslant n^{p}\left(\|f\|_{H^{p}(B)}^{p}-|f(0)|^{p}\right)
\end{aligned}
$$

which shows Lemma 3.

In the proof of the rest of this paper, we use the same letter $C$ to denote a positive constant which may change with each occurrence.

Lemma 4. Let $G(z, a)$ and $g(z)$ be as defined in Section 2. Then there exists a positive constant $C$ such that

$$
\int_{B} \frac{G(z, a)}{1-|a|^{2}} d V(a) \leqslant C g(z)
$$

Proof: Since $\left|\varphi_{a}(z)\right|=\left|\varphi_{z}(a)\right|$, we have

$$
\int_{B} \frac{G(z, a)}{1-|a|^{2}} d V(a)=\int_{B} \frac{g\left(\varphi_{a}(z)\right)}{1-|a|^{2}} d V(a)=\int_{B} \frac{g\left(\varphi_{z}(a)\right)}{1-|a|^{2}} d V(a) .
$$

By making the change of variable $\varphi_{z}(a)=w$ and using formulas (1) and (2), we see that the last integral becomes

$$
\left(1-|z|^{2}\right)^{n} \int_{B} \frac{g(w)}{\left(1-|w|^{2}\right)|1-\langle w, z\rangle|^{2 n}} d V(w)=\left(1-|z|^{2}\right)^{n}(I+I I)
$$

where I is the integral over $|w| \geqslant 1 / 2$ and II is the integral over $|w|<1 / 2$. It remains to show that each integral of I and II in (11) is bounded.

On $|w| \geqslant 1 / 2$, we clearly have $g(w) \leqslant C\left(1-|w|^{2}\right)^{n}$. Thus

$$
I \leqslant C \int_{|w| \geqslant 1 / 2} \frac{\left(1-|w|^{2}\right)^{n-1}}{|1-\langle w, z\rangle|^{2 n}} d V(w) \leqslant C \int_{B} \frac{\left(1-|w|^{2}\right)^{n-1}}{|1-\langle w, z\rangle|^{2 n}} d V(w) .
$$

Therefore the first integral $\mathrm{I}$ is bounded by Proposition 1.4.10 in [4]. 
For the second integral, it is easy to verify that

$$
\mathrm{II} \leqslant C \int_{|w|<1 / 2} g(w) d V(w) \leqslant C \int_{|w|<1 / 2} \frac{1}{|w|^{2 n-1}} d V(w)
$$

and integrating in polar coordinates the last integral shows that the second integral II is bounded. This finishes the proof.

We now turn to the proof of the main result of this paper.

Proof of тheorem: Suppose $f$ is analytic on $B$. By successive applications of $(8),(4)$ and (5) in this order, we have

$$
\begin{aligned}
{\left[\tilde{\triangle}|f|^{2}(0)\right]^{p / 2}=|\nabla f(0)|^{p} } & \leqslant C \int_{S}|f(\xi)|^{p} d \sigma-|f(0)|^{p} \\
& \leqslant C \int_{B}\left(\tilde{\triangle}|f|^{p}\right)(w) g(w) d \tau(w) \\
& =C \int_{B}|f(w)|^{p-2}\left(\tilde{\triangle}|f|^{2}\right)(w) g(w) d \tau(w) .
\end{aligned}
$$

If $f$ is replaced by $f \circ \varphi_{a}$, we obtain by a simple change of variables

$$
\begin{aligned}
{\left[\widetilde{\Delta}|f|^{2}(a)\right]^{p / 2} } & \leqslant C \int_{B}\left|f \circ \varphi_{a}(w)\right|^{p-2}\left(\widetilde{\triangle}|f|^{2}\right)\left(\varphi_{a}(w)\right) g(w) d \tau(w) \\
& =C \int_{B}|f(z)|^{p-2}\left(\widetilde{\triangle}|f|^{2}\right)(z) G(z, a) d \tau(z)
\end{aligned}
$$

If we now integrate both sides of (12) with respect to the measure $d V(a) /\left(1-|a|^{2}\right)$ and use (4), (5) and (10), we obtain

$$
\begin{aligned}
\int_{B}\left[\tilde{\Delta}|f|^{2}(a)\right]^{p / 2} \frac{d V(a)}{1-|a|^{2}} & \leqslant C \int_{B}|f(z)|^{p-2}\left(\tilde{\Delta}|f|^{2}\right)(z)\left\{\int_{B} \frac{G(z, a)}{1-|a|^{2}} d V(a)\right\} d \tau(z) \\
& \leqslant C \int_{B}|f(z)|^{p-2}\left(\tilde{\Delta}|f|^{2}\right)(z) g(z) d \tau(z) \\
& \leqslant C\|f\|_{H^{p}(B)}^{p}
\end{aligned}
$$

Therefore our conclusion comes easily from (6).

\section{REFERENCES}

[1] J.S. Choa and B.R. Choe, 'A Littlewood-Paley type identity and a characterization of BMOA', Complex Variables Theory Appl. 17 (1991), 15-23. 
[2] J.E. Littlewood and R.E.A.C. Paley, 'Theorems on Fourier series and power series. II', Proc. London Math. Soc. 42 (1936), 52-89.

[3] D.H. Luecking, 'A new proof of an inequality of Littlewood and Paley', Proc. Amer. Math. Soc. 103 (1988), 887-893.

[4] W. Rudin, Function theory in the unit ball of $\mathbf{C}^{\mathbf{n}}$ (Springer-Verlag, Berlin, Heidelberg, New York, 1980).

[5] D. Ullrich, 'Radial limits of $M$-subharmonic functions', Trans. Amer. Math. Soc. 292 (1985), 501-518.

Department of Mathematics Education

Sung Kyun Kwan University

Seoul 110-745

Korea
Department of Mathematics

Korea Advanced Institute of

Science and Technology

Taejon 305-701

Korea 